\title{
Malaria rapid diagnostic test positivity rate among febrile patients seen at the Paediatric emergency unit of a tertiary care facility
}

\author{
${ }^{* 1}$ Obu, D. C., ${ }^{1}$ Asiegbu, U. V., ${ }^{1}$ Okereke, B. E., ${ }^{1}$ Ukoh, U. C., ${ }^{2}$ Ujunwa, F. A., ${ }^{1}$ Afefi, C. O., \\ ${ }^{1}$ Enya, V. E., ${ }^{1}$ Item, S., and ${ }^{3}$ Efunshile, A. M.
}

${ }^{1}$ Department of Paediatrics, Alex Ekwueme Federal University Teaching Hospital Abakaliki, Ebonyi State, Nigeria ${ }^{2}$ Department of Paediatrics, University of Nigeria, Enugu State, Nigeria

${ }^{3}$ Department of Clinical Microbiology, Alex Ekwueme Federal University Teaching Hospital Abakaliki, Ebonyi State, Nigeria

*Correspondence to: obudora@yahoo.com; +2348037511272

\begin{abstract}
:
Background: Malaria, a life-threatening parasitic disease transmitted to humans by the female Anopheles mosquito is one of the infectious causes of fever in children. In Nigeria, malaria remains one of the most important health problems, accounting for $25 \%$ of infants and $30 \%$ of under-five mortalities. The objective of this study was to determine the prevalence of malaria among febrile children presenting at the children's emergency room (CHER) of a tertiary health facility in Abakaliki using a malaria rapid diagnostic test (mRDT). Methodology: This was a retrospective study that involved children presenting with fever in CHER over a 3-year period. A total of 1,273 febrile children below 18 years of age were tested with a malaria rapid diagnostic test (mRDT) kit during this period. Medical records of the patients were assessed to retrieve information such as age, gender, and clinical diagnoses. Data were analyzed using SPSS version 25.

Results: A total of 707 (55.5\%) were males and $883(69.4 \%)$ were below 5 years of age. The overall prevalence of malaria by the mRDT test was $26 \%(n=331)$. Uncomplicated malaria, $283(22.2 \%)$ was the commonest clinical diagnosis made while the least was malnutrition $3(0.2 \%)$. Children aged $10-<18$ years were predominantly affected as mRDT positivity rate was significantly higher in children age group $10-<18$ years $(40.4 \% 76 / 189)$ than other age groups $\left(X^{2}=44.76, p<0.001\right)$. Similarly, the rate was significantly higher (OR $9.625,95 \%$ CI $7.233-12.808, p<0.0001)$ in children with the clinical diagnosis of malaria $(55.2 \%, 235 / 426)$ than those with the clinical diagnosis of other illnesses (11.3\%, 96/847), and significantly higher (OR $0.19,95 \%$ CI $0.1186-0.3043$, $p<0.0001)$ among those clinically diagnosed with complicated $(79.7 \%, 114 / 143)$ than those with uncomplicated malaria $(42.8 \%, 121 / 283)$.

Conclusion: There is a high prevalence of malaria among febrile children presenting at the CHER of Alex Ekwueme Federal University Teaching Hospital Abakaliki. Children age group $10-<18$ years were predominantly affected. The use of mRDT should be encouraged both as a screening and diagnostic tool with a protocol such that febrile children who have positive results are confirmed as having malaria while those with negative results are further evaluated with microscopy.
\end{abstract}

Keywords: children; malaria; prevalence; rapid diagnostic test; emergency room

Received Aug 5, 2021; Revised Oct 18, 2021; Accepted Oct 19, 2021; Published online Jan 18, 2022

Copyright 2022 AJCEM Open Access. This article is licensed and distributed under the terms of the Creative Commons Attrition 4.0 International License <a rel="license" href="http://creativecommons.org/licenses/by/4.0/", which permits unrestricted use, distribution and reproduction in any medium, provided credit is given to the original author(s) and the source. Editor-in-Chief: Prof. S. S. Taiwo

\section{Taux de positivité des tests de diagnostic rapide du paludisme chez les patients fébriles vus à l'unité d'urgence pédiatrique d'un établissement de soins tertiaires}

\author{
${ }^{* 1}$ Obu, D. C., ${ }^{1}$ Asiegbu, U. V., ${ }^{1}$ Okereke, B. E., ${ }^{1}$ Ukoh, U. C., ${ }^{2}$ Ujunwa, F. A., ${ }^{1}$ Afefi, C. O., \\ ${ }^{1}$ Enya, V. E., ${ }^{1}$ Item, S., et ${ }^{3}$ Efunshile, A.M.
}
${ }^{1}$ Département de Pédiatrie, Hôpital universitaire fédéral Alex Ekwueme d'Abakaliki, État d'Ebonyi, Nigéria ${ }^{2}$ Département de Pédiatrie, Université du Nigéria, État d'Enugu, Nigéria
${ }^{3}$ Département de microbiologie clinique, Hôpital universitaire fédéral Alex Ekwueme d'Abakaliki, État d'Ebonyi, Nigéria
*Correspondance à: obudora@yahoo.com; +2348037511272




\section{Résumé:}

Contexte: Le paludisme, une maladie parasitaire mortelle transmise à I'homme par le moustique femelle Anopheles, est l'une des causes infectieuses de fièvre chez les enfants. Au Nigeria, le paludisme reste l'un des problèmes de santé les plus importants, représentant $25 \%$ des nourrissons et $30 \%$ des décès des moins de cinq ans. L'objectif de cette étude était de déterminer la prévalence du paludisme chez les enfants fébriles se présentant à la salle d'urgence pour enfants (CHER) d'un établissement de santé tertiaire à Abakaliki à l'aide d'un test de diagnostic rapide du paludisme (mRDT).

Méthodologie: Il s'agit d'une étude rétrospective portant sur des enfants présentant de la fièvre en CHER sur une période de 3 ans. Au total, 1273 enfants fébriles de moins de 18 ans ont été testés avec un kit de test de diagnostic rapide du paludisme (mRDT) au cours de cette période. Les dossiers médicaux des patients ont été évalués pour récupérer des informations telles que l'âge, le sexe et les diagnostics cliniques. Les données ont été analysées à l'aide de SPSS version 25.

Résultats: Au total, $707(55,5 \%)$ étaient des hommes et $883(69,4 \%)$ avaient moins de 5 ans. La prévalence globale du paludisme par le test mRDT était de $26 \%(n=331)$. Le paludisme non compliqué, $283(22,2 \%)$ était le diagnostic clinique le plus fréquemment posé alors que le moins était la malnutrition $3(0,2 \%)$. Les enfants âgés de 10 à $<18$ ans étaient principalement touchés, car le taux de positivité du mRDT était significativement plus élevé chez les enfants du groupe d'âge $10-<18$ ans $(40,4 \% 76 / 189)$ que les autres groupes d'âge $\left(X^{2}=44,76\right.$, $p<0,001)$. De même, le taux était significativement plus élevé (OR 9,625, IC à 95\% 7,233-12,808, $p<0,0001$ ) chez les enfants ayant reçu un diagnostic clinique de paludisme $(55,2 \%, 235 / 426)$ que ceux ayant reçu un diagnostic clinique d'autres maladies $(11,3 \%, 96 / 847$ ), et significativement plus élevés (OR 0,19, IC à 95\% $0,1186-0,3043, p<0,0001)$ parmi les patients cliniquement diagnostiqués avec un paludisme compliqué $(79,7 \%$, $114 / 143)$ que ceux avec un paludisme non compliqué $(42,8 \%, 121 / 283)$.

Conclusion: Il existe une prévalence élevée de paludisme chez les enfants fébriles se présentant au CHER du CHU Alex Ekwueme d'Abakaliki. Les enfants du groupe d'âge 10-<18 ans étaient principalement touchés. L'utilisation du mRDT devrait être encouragée à la fois comme outil de dépistage et de diagnostic avec un protocole tel que les enfants fébriles qui ont des résultats positifs sont confirmés comme ayant le paludisme tandis que ceux qui ont des résultats négatifs sont évalués plus avant par microscopie.

Mots-clés: enfants; paludisme; prévalence; test de diagnostic rapide; urgences

\section{Introduction:}

Febrile illnesses in children are worrisome to caregivers and are one of the major reasons for hospital visits in the Paediatric department in developing countries. At times, it creates anxiety among caregivers leading to presentation to the Children's emergency room (CHER) (1). Infectious causes of febrile illnesses could be viral, bacterial, fungal, or parasitic agents. Malaria a life-threatening disease caused by the plasmodium parasite which is transmitted to humans by the female Anopheles mosquito is present in 99 countries and territories. The disease is of serious public health concern in sub-Saharan Africa especially among children and pregnant women (2). Children under five years of age are highly vulnerable to malaria and face dire consequences such as severe malaria if they are not promptly and adequately treated (3).

The 2019 world malaria report indicated that 228 million malaria cases and 405,000 malaria deaths occurred worldwide. A documented $93 \%$ of the estimated cases and $94 \%$ of the global malaria deaths occurred in the African continent. Nigeria accounts for the highest burden of malaria cases and death (4). It is estimated that about $25 \%$ of the global malaria cases and $24 \%$ of all malaria deaths occurred in Nigeria in 2018 (4). Children less than five years were the most vulnerable accounting for $67 \%(272,000)$ of global malaria deaths. In Nigeria, malaria remains one of the most important health problems, accounting for $25 \%$ of infant mortality, $30 \%$ of under five mortality, and $11 \%$ of maternal mortality (5).

One of the key malaria control strategies in an area with a high burden of malaria is early diagnosis and prompt and effective treatment of childhood fevers caused by malaria (6). The signs and symptoms obtained from patients by physicians play a key role in the clinical diagnosis of malaria. Clinical diagnosis is still used for the therapeutic care of most febrile individuals by physicians in several malaria-endemic regions despite some reported cases of its imprecision as early malarial clinical features vary and are nonspecific hence, its sole utilization becomes quite challenging and unreliable (7). Therefore, clinical diagnosis of malaria without laboratory support may lead to misdiagnosis and wrong treatment (8).

The Nigerian malarial control policy recommends universal diagnostic testing by microscopy or malaria rapid diagnostic tests in all suspected cases and the use of artemisininbased combination therapy (ACT) for the treatment of malaria (9). Microscopy requires a laboratory setup and a trained microscopist, and constant availability of electricity which limits its applicability as both requirements are lacking in many health care settings. In these settings, therefore, the malaria rapid diagnostic test (mRDT) provides a parasite-based rapid, early, and accurate diagnosis of malaria (10). It requires a few drops of blood, little expertise, and takes only 5-15 min to perform. It is thus suitable for usage in malaria-endemic areas, settings with limited health perso- 
nnel and facilities, and during outbreaks. Its usage has been reported to significantly reduce referrals and inpatient length of hospital stay (11).

Despite efforts of the malaria control program in the past decade, malaria remains a primary cause of morbidity and mortality globally (12). Studies have shown malaria prevalence of $30.5 \%$ and $83.1 \%$ in Nigerian children $(13,14)$. Onwe et al., (15) once studied the prevalence of malaria parasitemia among children aged $0-15$ years in Abakaliki using microscopy as a diagnostic method and reported a prevalence rate of $24.1 \%$. The present study was aimed to determine the prevalence of malaria among febrile children presenting at CHER of Alex Ekwueme Federal University Teaching Hospital Abakaliki (AEFUTHA) to provide updated data on malaria epidemiology and surveillance.

\section{Materials and method:}

\section{Study location and setting}

The study was conducted at the Alex Ekwueme Federal University Teaching Hospital Abakaliki, a tertiary institution created by a merger of the former Federal Medical Center Abakaliki and Ebonyi State University Teaching Hospital Abakaliki to a Federal Teaching Hospital in December 2011. It is one of the tertiary health facilities located in Abakaliki metropolis, Ebonyi State. Ebonyi State belongs to the southeast geopolitical zone bounded in the north by Benue State, in the west by Enugu State, in the east by Cross River State, and in the south by Abia State. It occupies a land area of 5,953 square kilometers and situated between longitude $7^{\circ} 30^{\prime}$ and $8^{\circ}$ $30^{\prime} \mathrm{E}$ and between latitude $5^{\circ} 40^{\prime}$ and $6^{\circ} 54^{\prime} \mathrm{N}$.

The vegetation of the State is predominately tropical rainforest with a mean annual rainfall of $2100 \mathrm{~mm}$. The rainy season starts from April to October and the dry season from October to March (16). The state lies entirely in the Cross River plains with frequent floods during the rainy season, resulting from poor drainage systems, stagnant streams, and ponds that predispose the state to the menace of mosquito infestations and malaria endemicity. About $75 \%$ of the state population is engaged in subsistent agrarian economic activities especially rice farming which also produces breeding sites for mosquitoes (16).

\section{Study design and ethical approval}

This was a retrospective study of febrile children who presented in CHER from November 2016 to October 2019. A total of 1,273 children under the age of 18 years out of 5,568 who attended the CHER with a history of fever were tested for malaria with a finger prick mRDT kit. Ethical approval for the study was obtained from the Human Research and Ethical Committee of the hospital.

\section{Malaria rapid diagnostic test}

Parasitological diagnosis of malaria was done in-house in the Paediatrics department's side laboratory using the SD Bioline Malaria Ag P.f (HRP-II) ${ }^{\mathrm{TM}}$ device (05FK50 Standard Diagnostics, Inc. Gyeonggi-do, Republic of Korea). This is a rapid qualitative test based on lateral flow immunochromatography in cassette format which detects Plasmodium falciparum-specific protein and histidine-rich protein II (HRP-II) antigen in human whole blood. The device contains a membrane strip that is pre-coated with mouse monoclonal antibodies specific for HRP-II of P.f-colloid gold conjugate that reacts with $P$. falciparum antigen in the specimen. The antigen in the blood moves along the membrane chromatographically to the test region ' $P$. $f$ ' and forms a visible line as the antibody-antigen-antibody gold particle complex. The manufacturer's performance ratings of the kit for detecting $P$. falciparum in blood are sensitivity of $99.7 \%$ and specificity of $99.5 \%$.

The protocol for testing was contained in a standard operating procedure (SOP) and a bench aid was always pasted on the wall of the laboratory for a quick reference. About $5 \mu \mathrm{l}$ of blood, collected aseptically through a finger prick from each patient, was transferred to the sample well of the mRDT cassette while drops of chase buffer were applied to the other well in line with the manufacturer's instruction. Test results were interpreted after the recommended waiting period of 15 minutes. A test was interpreted to be positive if both control and patients' sample lines turn red, and negative if only the control line turned red. Results were interpreted to be invalid if the patient's test line turned red, but the control line was not visible. Invalid results were repeated immediately with another kit. Internal quality control was carried out on each batch of mRDT kit supplied to the laboratory by using malaria-positive blood samples from clinical cases that were also confirmed by microscopy.

\section{Data collection}

The medical registers of data of all children who presented with fever and who had mRDT tests done were retrieved. Information obtained included age, gender, and clinical diagnoses by admitting physicians, and the results of mRDT conducted on the patients. Clinical diagnosis of malaria was based on the presence of features of malaria such as the history of fever or presence of elevated temperature, vomiting, loss of appetite, body weakness, bitter taste in the mouth, and others. Confirmation of malaria was done by 
the presence of a positive mRDT in such study participants.

\section{Statistical analysis:}

Data analysis was performed using the SPSS ${ }^{\circ}$ Statistics program, version 25 (IBM Corporation, Armonk, NY, USA). Descriptive statistics (frequency and percentages) were calculated for categorical variables. Chi-square test was used to determine the association between mRDT test result and patients' variables. A p-value of $<0.05$ was considered statistically significant.

\section{Results:}

Socio-demographic characteristics of study participants:

A total of $1,273(22.8 \%)$ febrile children out of 5,568 children that presented at the CHER during the 3-year study period were tested for the presence of malaria parasite $(P$. falciparum) using mRDT kit. Seven hundred and seven (55.5\%) were males and 883 $(69.4 \%)$ were below 5 years of age (Table 1$)$.

Table 1: Socio-demographic characteristics of the study participants

\begin{tabular}{cc}
\hline Variables & Frequency (\%) \\
\hline Gender & $707(55.5)$ \\
Male & $566(44.5)$ \\
Female & \\
Age group (years) & $883(69.4)$ \\
$0-<5$ & $201(15.80)$ \\
$5-<10$ & $189(14.8)$ \\
$10-<18$ & $\mathbf{1 2 7 3 ( 1 0 0 )}$ \\
\hline Total
\end{tabular}

\section{Clinical diagnosis in febrile children presenting in CHER}

The commonest initial clinical diagnosis made among the febrile children presenting in CHER was malaria $(33.4 \%, 426$ / $1273)$, with uncomplicated malaria $(22.2 \%$, $283 / 1273$ ) and complicated malaria (11.2\%, $143 / 1273)$, while the least common diagnosis was malnutrition $(0.2 \%, 3 / 1273)$. Other diagnoses included malignancies, otitis media, syncope, helminthiasis, food poisoning, viral hepatitis, and diabetic ketoacidosis) (Table 2).

Table 2: Clinical diagnosis of the study participants

\begin{tabular}{cc}
\hline Variable & Frequency (\%) \\
\hline Uncomplicated malaria & $283(22.2)$ \\
Complicated malaria & $143(11.2)$ \\
Gastroenteritis & $238(18.7)$ \\
Upper respiratory tract & $151(11.9)$ \\
infection & $85(6.7)$ \\
Sepsis & $42(3.3)$ \\
Bronchopneumonia & $42(3.3)$ \\
Sickle cell crisis & $30(2.4)$ \\
Febrile convulsion & $22(1.7)$ \\
Enteric fever & $17(1.3)$ \\
Meningitis & $13(1.0)$ \\
Urinary tract infection & $13(1.0)$ \\
Heart failure & $8(0.6)$ \\
Asthma & $8(0.6)$ \\
Shock & $5(0.4)$ \\
Anaemia & $3(0.2)$ \\
Malnutrition & $170(13.4)$ \\
Others & $\mathbf{1 2 7 3 ( 1 0 0 . 0 )}$ \\
\hline Total &
\end{tabular}

Result of malaria RDT in febrile children

A total of 331 (26\%) febrile children with a clinical diagnosis of malaria (complicated or uncomplicated) tested positive to mRDT (Table 3). The mRDT was positive in $55.2 \%(235 / 426)$ of all the children with malaria, [42.8\% $(121 / 283)$ in children with uncomplicated malaria was significantly lower than $79.7 \%(114 / 143)$ in children with complicated malaria (OR 0.19, 95\% CI 0.1186$0.3043, p<0.0001)]$. The positivity rate of mRDT was also significantly higher (OR 9.625, $95 \%$ CI 7.233-12.808, $p<0.0001$ ) in children with a diagnosis of malaria (55.2\%, 235/426) than in children with a diagnosis of other illnesses (11.3\%, 96/847)

For the age group of the children, mRDT positivity rate was significantly higher $\left(X^{2}=44.76, p<0.0001\right)$ in the age group 10 $<18$ years $(40.4 \%, 76 / 189)$ than in the age groups $5-<10$ years $(36.3 \%, 73 / 201)$ and 0 $<5$ years $(20.6 \%, 182 / 883)$. However, mRDT positivity rate was not significantly different between males $(25 \%, 177 / 707)$ and females (27.2\%, 154/566) children (OR 0.8935, 95\% CI $0.6948-1.149, p=0.4156)$. 
Table 3: Association between age, gender, clinical diagnosis, and outcome of malaria Rapid diagnostic test

\begin{tabular}{|c|c|c|c|c|}
\hline \multirow[t]{2}{*}{ Variable } & \multicolumn{2}{|c|}{$\begin{array}{c}\text { Results of mRDT } \\
n(\%)\end{array}$} & \multirow[t]{2}{*}{$X^{2}$} & \multirow[t]{2}{*}{$p$ value } \\
\hline & Positive & Negative & & \\
\hline \multicolumn{5}{|l|}{ Age group (years) } \\
\hline $0-<5$ & $182(20.6)$ & $701(79.4)$ & 44.76 & $<0.001 *$ \\
\hline $5-<10$ & $73(36.3)$ & $128(63.7)$ & & \\
\hline $10-<18$ & $76(40.4)$ & $112(59.6)$ & & \\
\hline \multicolumn{5}{|l|}{ Gender } \\
\hline Male & $177(25.0)$ & $530(75.0)$ & 0.6627 & 0.4156 \\
\hline Female & $154(27.2)$ & $412(72.8)$ & & \\
\hline \multicolumn{5}{|l|}{ Initial clinical diagnosis } \\
\hline Uncomplicated malaria & $121(9.5)$ & $162(12.7)$ & 384.53 & $<0.001) *$ \\
\hline Complicated malaria & $114(9.0)$ & $29(2.3)$ & & \\
\hline $\begin{array}{l}\text { Upper respiratory tract } \\
\text { infection }\end{array}$ & $15(1.2)$ & $136(10.7)$ & & \\
\hline Febrile convulsion & $14(1.1)$ & $16(1.3)$ & & \\
\hline Gastroenteritis & $12(0.9)$ & $226(17.8)$ & & \\
\hline Sepsis & $12(0.9)$ & $73(5.7)$ & & \\
\hline Sickle cell crisis & $6(0.5)$ & $36(2.8)$ & & \\
\hline Meningitis & $5(0.4)$ & $12(0.9)$ & & \\
\hline Enteric fever & $4(0.3)$ & $18(1.4)$ & & \\
\hline Bronchopneumonia & $3(0.2)$ & $39(3.1)$ & & \\
\hline Heart failure & $3(0.2)$ & $10(0.8)$ & & \\
\hline Urinary tract infection & $3(0.2)$ & $10(0.8)$ & & \\
\hline Malnutrition & $1(0.08)$ & $20.2)$ & & \\
\hline Shock & $0(0.0)$ & $8(0.6)$ & & \\
\hline Asthma & $0(0.0)$ & $8(0.6)$ & & \\
\hline Anaemia & $0(0.0)$ & $5(0.4)$ & & \\
\hline Others & $18(1.4)$ & 152 (11.9) & & \\
\hline Total & $331(26.0)$ & $932(74.0)$ & & \\
\hline
\end{tabular}

* statistically significant

\section{Discussion:}

The study revealed an overall malaria prevalence of $26.0 \%$ among febrile cases seen in CHER. This is not unexpected because our study area is in a malaria-endemic region. Also, Abakaliki is a rice-producing center in Nigeria where farmers deliberately flood farmlands to enhance rice yields. This practice is known to enhance mosquito breeding (17). This was supported by the findings of Onwe et al. (15) who also found a prevalence of $24.1 \%$ in an earlier study. Another study in the northern region of Nigeria where rice is also produced, reported a similar prevalence of $26.4 \%$ (18). The finding was comparable to reports of $22.1 \%$ in Ghana (19) and $22.0 \%$ in Gabon (1) but lower than $75.77 \%$ in Benin (20) and $83.1 \%$ in Imo State (14). A study conducted in India reported a prevalence of $36.6 \%(21)$. These variations in malaria prevalence may be due to the age of study participants, diagnostic methods, and differences in sensitivities of mRDT kits used. The studies in Nigeria, Benin, and India involved only under 5 children who are more prone to malaria attack and may have accounted for the high prevalence reported $(18,20,21)$. 
The present study alongside studies done in India used mRDT for diagnosis which serves as a screening tool with varying sensitivity and specificity to malaria parasites while other studies done in Abuja, and Anambra States in Nigeria used microscopy which is regarded as the 'gold standard' test for malaria diagnosis. Different socioeconomic statuses of study participants and/or plasmodial transmission rates, as well as a decrease in free distribution of impregnated bed nets in addition to its utilization and awareness campaigns on malaria, are also possible contributors. This emphasizes the need to intensify the usage of indoor residual spray, insecticidetreated nets, and other interventions to sustain a reduction in transmission of malaria parasites and the burden of malaria as this study showed an obvious decline in malaria prevalence rate among children.

In this study, children age group 10$<18$ years had the highest prevalence of malaria, with $40.4 \%$ positivity for $P$. falciparum on mRDT. This may be attributed to repeated exposure to the parasite in these children due to frequent mosquito bites caused by staying out late at night and playing with their body exposed most times. This observation agrees with the findings of a study conducted in the same locale (16). On the contrary, the findings of researchers in different parts of the world reported age groups below five years to be most affected $(1,22-24)$. Differences in childrearing practices and sociocultural factors may account for the variation. Gender was not associated with malaria positivity in our study, which agrees with reports obtained by other researchers $(23,25,26)$. However, some studies conducted in Benin reported that males had a significantly higher risk of malaria (20, 24).

There was significant association between clinical diagnosis and the result of mRDT in the sense that mRDT positivity rate was significantly higher $(p<0.0001)$ in children who had clinical diagnosis of malaria $(55.2 \%)$ than those who had clinical diagnosis of other illnesses $(11.2 \%)$, and was also significantly higher $(p<0.0001)$ among those clinically diagnosed with complicated malaria $(79.7 \%)$ than those with uncomplicated malaria (42.8\%). This suggests a reliable clinical sense of judgement about the ability of clinicians to diagnose malaria, and may also indicate increased diagnostic sensitivity of mRDT in complicated malaria, probably from increased parasite antigens from heavy parasitaemia. However, because of the possibility of missed diagnosis of uncomplicated malaria in some children which may progress to complicated malaria with a higher risk of mortality, such febrile children should benefit from mRDT testing. Some researchers had observed that mRDTs had significantly higher sensitivity and diag- nostic accuracy than clinical diagnosis (27). Therefore, mRDTs should be used to complement microscopy or used alone when expert microscopy is unavailable.

Our study was limited by the fact that it was a retrospective analysis of records, as such, the information obtained was more limited and could not show determinants of malaria among study participants. Furthermore, the accuracy of the mRDTs and results generated could have been affected by several factors including transport and storage of kits and parasite density of a sample.

\section{Conclusion:}

This study showed a high prevalence of malaria among febrile children seen in the Children Emergency Room (CHER) of Alex Ekwueme Federal University Teaching Hospital, Abakaliki (AEFUTHA). Children age group $10-<18$ years were predominantly affected. Malaria rapid diagnostic test positivity was significantly associated with clinical diagnosis of malaria than clinical diagnosis of other illnesses, and was more sensitive in the diagnosis of complicated than uncomplicated malaria. The use of mRDT should be encouraged both as a screening and diagnostic tool with a protocol such that febrile children who have positive results are confirmed as having malaria while those with negative results are further evaluated with microscopy.

\section{Acknowledgments:}

The authors appreciate the suppliers of mRDT kits to the facility and are grateful to parents/caregivers as well as the study participants.

\section{Conflict of interest:}

Authors declare no conflict of interest

\section{References:}

1. Maghendji-Nzondo, S., Nzoughe, H., Lemamy, G. J., et al. Prevalence of malaria, prevention measures, and main clinical features in febrile children admitted to the Franceville Regional Hospital, Gabon. Parasite. 2016; 23: 32

doi: 10.1051/parasite/2016032.

2. Alvarez, J. R., Al-Khan, A., and Apuzzio, J. J. Malaria in pregnancy. Infect Dis Obstet Gynecol. 2005; 13 (4): 229-236.

3. Owusu-Agyei, S., Asante, K. P., Adjuik, M., et al. Epidemiology of malaria in the forest-savanna transitional zone of Ghana. Malar J. 2009; 8 (1): 220.https://doi.org/10.1186/1475-2875-8-220.

4. World Health Organization. World Malaria Report 2019.https://www.who.int/publications-detailredirect/9789241565721 (accessed Jul 19, 2021)

5. Nigeria Malaria Indicator Survey [MIS20]. 2015. https://dhsprogram.com/pubs/pdf/MIS20/MIS20.p df (accessed Jul 20, 2021)

6. Lee, J-H., Jang, J. W., Cho, C. H., et al. FalsePositive Results for Rapid Diagnostic Tests for Malaria in Patients with Rheumatoid Factor. J Clin Microbiol. 2014; 52 (10): 3784-3787. 
7. McMorrow, M. L., Masanja, M. I., Abdulla, S. M. K., Kahigwa, E., and Kachur, S. P. Challenges in Routine Implementation and Quality Control of Rapid Diagnostic Tests for Malaria-Rufiji District, Tanzania. Am J Trop Med Hyg. 2008; 79 (3): 385-390

8. Oladosu, O. O., and Oyibo, W. A. Overdiagnosis and Overtreatment of Malaria in Children that Presented with Fever in Lagos, Nigeria. ISRN Infect Dis. 2012; 2013:1-6.

9. Guidelines for the Treatment of Malaria. 2nd ed. Geneva: World Health Organization; 2010.

http://www.ncbi.nlm.nih.gov/books/NBK254223/ (accessed Jul 24, 2021)

10. Wernsdorfer, W. H., Muth, S., Wongsrichanalai, C., Sutamihardja, A., and Barcus, M. J. A Review of Malaria Diagnostic Tools: Microscopy and Rapid Diagnostic Test (RDT). Am J Trop Med Hyg. 2007; 77 (6_Suppl):119-127.

11. Boyce, R.M., Muiru, A., Reyes, R., et al. Impact of rapid diagnostic tests for the diagnosis and treatment of malaria at a peripheral health facility in Western Uganda: an interrupted time series analysis. Malar J. 2015; 14: 203 https://doi:10.1186/s12936-015-0725-0.

12. Wassmer, S. C., and Grau, G. E. R. Severe malaria: what's new on the pathogenesis front? Int Parasitol. 2017; 47 (2-3):145-152.

13. Gobir, Z., and Tukur, Z. Prevalence of malaria parasitemia using rapid diagnostic test among apparently healthy children in Kano, Nigeria. J Med Trop. 2014; 16 (1): 1-4.

14. Austin, N., Adikaibe, E., Ethelbert, O., Chioma, U., and Ekene, N. Prevalence and Severity of Malaria Parasitemia among Children Requiring Emergency Blood Transfusion in a Tertiary Hospital in Imo State, Nigeria. Ann Med Health Sci Res. 2014;4 (4): 619623.

15. Onwe, S. O., Mbamalu, C., Ani, O. C., Anthony, I., Nwovu, A. I., and Igwe, C. C. Prevalence of Malaria Parasitaemia among Paediatrics in Federal Teaching Hospital Abakaliki, Ebonyi State. 2018; 26 (4): 418-423.

16. Uneke, C., and Ibeh, L. Impacts of deforestation on malaria in south-eastern Nigeria: the epidemiological, socioeconomic, and ecological implications. Internet J Third World Med. 2008; 8 (1). https://doi:ispub.com/IJTW/8/1/4609.

17. Diuk-Wasser, M. A., Toure, M. B., Dolo, G., et al. Vector abundance and malaria transmission in ricegrowing in Mali. Am J Trop Med Hyg. 2005; 72 (6): 725-731.
18. Abdulkadir, I., Rufai, H. A., Ochapa, S. O., et al. Malaria rapid diagnostic test in children: The Zamfara, Nigeria experience. Niger Med J. 2015; 56 (4): 278-282.

19. Orish, V. N., Ansong, J. Y., Anagi, I. B., Onyeabor, O. S., Sanyaolu, A. O., and Iriemenam, N. C. Malaria and associated co-morbidity in children admitted with fever manifestation in Western Ghana: A retrospective study. J Infect Dev Ctries. 2015; 9 (11): 1257-1263.

20. Akinbo, F. O., Okaka, C. E., Omoregie, R., and Igbinuwen, O. Prevalence of malaria and anaemia among HIV- infected patients in Benin City, Nigeria. NZJ Med Lab Sci. 2009; 63(3):78-80.

21. Qureshi, I., Qureshi, M. A., Gudepu, R. K., and Arlappa, N. Prevalence of malaria infection among under five-year tribal children residing in malariaendemic forest villages. F1000Research. 2014; 3: 286.https://doi.org/10.12688/f1000research.5632.1.

22. Edelu, B. O., Ndu, I. K., Igbokwe, O. O., and Iloh, O. N. Severe falciparum malaria in children in Enugu, Southeast Nigeria. Nig J Clin Pract. 2018; 21 (10): 1349-1355.

23. Nmadu, P., Peter, E., Alexander, P., Koggie, A., and Maikenti, J. The Prevalence of Malaria in Children between the Ages 2-15 Visiting Gwarinpa General Hospital Life-Camp, Abuja, Nigeria. J HIth Sci. 2015; 5 (3):47-51.

24. Okafor, F. U., and Oko-Ose, J. N. Prevalence of malaria infections among children aged six months to eleven years (6 months-11 years) in a tertiary institution in Benin City, Nigeria. Glob Adv Res J Med Med Sci. 2012; 1 (10): 273-279

25. Umma, I., Robinson, W., Jamilu, F., and Gwarzo, G. Prevalence of malaria parasitaemia among febrile Nigerian children with severe malnutrition in Northwestern Nigeria. Nig J Basic Clin Sci. 2017; 14 (2): 113-116

26. Abossie, A., Yohanes, T., Nedu, A., Tafesse, W., and Damitie, M. Prevalence of Malaria and Associated Risk Factors Among Febrile Children Under Five Years: A Cross-Sectional Study in Arba Minch Zuria District, South Ethiopia. Infect Drug Resist. 2020; 13:363-372.

27. Wogu, M. N., and Nduka, F. O. Evaluating Malaria Prevalence Using Clinical Diagnosis Compared with Microscopy and Rapid Diagnostic Tests in a Tertiary Healthcare Facility in Rivers State, Nigeria. J Trop Med. 2018

https://www.hindawi.com/journals/jtm/2018/3954 717/ 